\title{
An International Survey of PET/CT Clinical Reporting
}

\author{
Katherine A. Zukotynski ${ }^{1}$, Ryan D. Niederkohr ${ }^{2}$, Bennett S. Greenspan ${ }^{3}$, John O. Prior ${ }^{4}$, Heiko Schöder ${ }^{5}$, Marc A. \\ Seltzer ${ }^{6}$, Eric M. Rohren ${ }^{7}$, and Don C. Yoo $^{8}$ \\ ${ }^{I}$ Department of Medicine and Radiology, McMaster University, Hamilton, Ontario, Canada; ${ }^{2}$ Department of Nuclear Medicine, \\ Kaiser Permanente Medical Center, Santa Clara, California; ${ }^{3}$ Consultant, North Augusta, South Carolina; ${ }^{4}$ Department of Nuclear \\ Medicine and Molecular Imaging, Lausanne University Hospital, Lausanne, Switzerland; ${ }^{5}$ Department of Radiology, Memorial Sloan \\ Kettering Cancer Center, New York, New York; ${ }^{6}$ Department of Radiology, Dartmouth-Hitchcock Medical Center, Lebanon, New \\ Hampshire; ${ }^{7}$ Department of Radiology, Baylor College of Medicine, Houston, Texas; and ${ }^{8}$ Department of Diagnostic Imaging, Warren \\ Alpert Medical School of Brown University, Providence, Rhode Island
}

\section{See the associated article on page 480.}

$\mathbf{T}$ he report for an oncologic PET/CT scan is the primary method by which the physician interpreting the scan communicates with the referring physician and health-care team. The content of this report provides information on disease extent, biologic characteristics, and therapy response, among other details. It ultimately directs patient care (1), serves as legal documentation of services provided and possible technical limitations (2), and, if clear and accurate, may mitigate issues associated with delayed cancer diagnosis, false-positive results, and incidental findings, among causes of malpractice litigation (3-5). Other reasons for a well-constructed report include billing and compliance, as well as education and research. Although pertinent positive or negative findings and report templates vary depending on the disease histology and site, there are key components that transcend contextual reporting. The Society of Nuclear Medicine and Molecular Imaging, American College of Radiology, and European Association of Nuclear Medicine have published practice guidelines listing key components of an oncologic PET/CT report (6-9): the clinical indication, the technique, comparison studies, findings, and the impression.

The clinical indication details the tumor histology, site, relevant history, and questions raised.

The technique includes the radiopharmaceutical name, activity, route of administration, uptake time (e.g., from injection to start of imaging), ancillary medications, adverse reactions, blood glucose, and CT parameters (including contrast use, breathing conditions, field of view, and deviations from standard protocol). Precise details are preferred, and the words approximate and nondiagnostic should be avoided. In some states or countries, CT parameters (e.g., kVp and $\mathrm{mAs}$ ) or patient radiation exposure (e.g., CT dose index in $\mathrm{mGy}$ or dose-length product in $\mathrm{mGy} \cdot \mathrm{cm}$ ) may be required by law.

Received Jan. 4, 2019; revision accepted Feb. 11, 2019.

For correspondence or reprints contact: Katherine A. Zukotynski, Departments of Medicine and Radiology, McMaster University, 1200 Main St. W., Room 1P11, Hamilton, ON L8N 3Z5, Canada.

E-mail:zukotynk@mcmaster.ca

COPYRIGHT (c) 2019 by the Society of Nuclear Medicine and Molecular Imaging. DOI: 10.2967/jnumed.118.223073
Comparison and correlative imaging studies with dates should be listed. If no prior imaging is available, this should be stated.

Findings should be organized, often by order of clinical relevance, anatomic site, or a hybrid format. The location, extent, and intensity of abnormal radiotracer activity should be correlated anatomically and reported with standard descriptors (e.g., RadLex [http://radlex.org]). Size may be given as a single transaxial measurement, although 2-3 orthogonal dimensions are preferred, and descriptors (short or long axis, image slice number) may be helpful. Comparison with prior measurements should be included, when possible. Descriptors of radiotracer uptake (e.g., mild, moderate, or intense) and semiquantitative measures (e.g., SUV) are both often included in a report. The method of calculating and reporting SUV (e.g., using lean body mass, $\mathrm{SUV}_{\text {max }}, \mathrm{SUV}_{\text {peak }}$ or SUV equivalent [when 2 different PET/CT devices are being used and calibrated using a sphere phantom]) is not standardized, and the technique used should be clear. Inclusion of a measure of activity in a reference region of interest such as liver or aortic blood pool is helpful. If this is outside the expected range, the interpreting physician is alerted to the presence of a technical issue (e.g., incorrect patient weight, incorrect dose of radiopharmaceutical, or perhaps a significant infiltration of radiopharmaceutical) that might compromise SUV measurements at disease sites.

The impression should answer the clinical question, be brief whenever possible, and interpret the findings by indicating whether the study is normal or abnormal and if or how the disease has changed. When additional imaging or intervention may change management, the change in management can be recommended. Urgent, emergent, or unexpected findings must be communicated to the referring physician or surrogate and the communication documented in the report (e.g., date of communication, time, to whom, and means). If not completed, this can be a cause of adverse outcome and liability. Also, reports are available to (and increasingly requested by) patients, and it should always be remembered that anxiety-provoking language can be problematic.

It has long been known that PET/CT reports vary in format, content, and quality. A review of reports from the National Oncologic PET Registry found key components (indication, comparison to prior imaging studies) were missing in over $40 \%$ of reports (10). In this issue of The Journal of Nuclear Medicine, the study by Freudenberg et al. (11) suggests not only an ongoing lack of adherence to standardized reporting but also a lack of awareness of existing PET/CT reporting guidelines by $30 \%$ of the respondents, with a further $15 \%$ not using them routinely. This snapshot of 
oncologic PET/CT reporting practice is based on the results of a web-based questionnaire with 242 evaluable responses, the majority from European specialists trained in radiology, nuclear medicine, or both. There are limitations to this study. Namely, since most responses were from Europe, it is somewhat unclear what the situation is on a more global basis. Further, since comparison to prior imaging is common, yet use of response criteria is not, it seems likely the evaluation of disease change conveyed to the referring clinician is based, at least in part, on a visual or subjective assessment. Finally, the results suggest not only a wide disparity in reporting style but also absence of reporting parameters that could affect the SUV for a given study, such as the uptake time. Certainly, reporting quality can be difficult to define and teach. However, when referring physicians receive high-quality reports, their confidence in the value of PET/CT increases, resulting in a greater impact on patient care.

In recent times, there has been a push toward standardization of reporting style and the use of criteria to clarify findings and standardize the impression of our reports. One should distinguish between inclusion of standardized elements and template reporting. Standardization does not imply the need for a checklist report and need not make the report longer or more difficult to read but, on the contrary, may help provide focus, allowing the findings to be tied together with the clinical question while including key component of a report. There is a host of criteria for baseline imaging and response assessment, most of which are of greatest importance for clinical trials (RECIST and PERCIST, among others). Outside of clinical trials, these criteria are generally not used in clinical practice. For example, in routine clinical oncology, there are no real-world clinical guidelines and standards directing the use of PERCIST measurements, and outside of clinical trials, PERCIST for PET/ $\mathrm{CT}$ is not routinely used in clinical practice. However, some imaging criteria (e.g., Deauville/Lugano) that encompass metabolic and anatomic imaging findings in patients with lymphoma are being incorporated into clinical practice and clinical guidelines (e.g., National Comprehensive Cancer Network) and are being used in routine clinical PET/CT reporting. Further, including all components of an examination in a single concise, unified report is becoming more pressing as hybrid imaging is ever more ubiquitous and is increasingly being used in the clinical algorithms for patient management. However, whereas standardized reporting has many advantages, there are also issues that remain. Perhaps the most important is the ongoing evolution in criteria used, suggesting perhaps that a version and reference for the criteria should also be included in our reports, lest the report become rapidly outdated. Although today there is no recommendation to include specific criteria into a PET/CT report, it seems likely such a recommendation is on our horizon.

To summarize, the content of an oncologic PET/CT report not only influences patient management and clinical outcomes but also is a legal documentation of services provided. The results of this paper suggest there is an unmet clinical need for education on oncologic PET/CT reporting. To ensure that our reports add value, we must strive for standardized high-quality reports. To achieve this goal, we recommend that specific metrics of what constitutes a high-quality standardized report (such as inclusion of the key components discussed in this perspective) be incorporated into existing institutional quality control processes, professional societal clinical practice guidelines, and site accreditation programs. The report metrics should be developed by joint consensus of imaging and referring physician specialists with expertise in $\mathrm{PET} / \mathrm{CT}$.

\section{DISCLOSURE}

No potential conflict of interest relevant to this article was reported.

\section{REFERENCES}

1. Naik SS, Hanbidge A, Wilson SR. Radiology reports: examining radiologist and clinician preferences regarding style and content. AJR. 2001;176:591598.

2. Thorwarth WT. Get paid for what you do: dictation patterns and impact on billing accuracy. J Am Coll Radiol. 2005;2:665-669.

3. Berlin L. The incidentaloma: a medicolegal dilemma. Radiol Clin North Am. 2011;49:245-255.

4. Brenner RJ, Bartholomew L. Communication errors in radiology: a liability cost analysis. J Am Coll Radiol. 2005;2:428-431.

5. Halpin SF. Medico-legal claims against English radiologists: 1995-2006. Br J Radiol. 2009;82:982-988.

6. Niederkohr RD, Greenspan BS, Prior JO, et al. Reporting guidance for oncologic ${ }^{18}$ F-FDG PET/CT imaging. J Nucl Med. 2013;54:756-761.

7. Delbeke D, Coleman RE, Guiberteau MJ, et al. Procedure guideline for tumor imaging with ${ }^{18}$ F-FDG PET/CT 1.0. J Nucl Med. 2006;47:885-895.

8. ACR-SPR practice guideline for performing FDG-PET/CT in oncology. ACR website. https://www.acr.org/-/media/ACR/Files/Practice-Parameters/fdg-pet-ct. pdf. Accessed February 27, 2019.

9. Boellaard R, Delgado-Bolton R, Oyen WG, et al. FDG PET/CT: EANM procedure guidelines for tumour imaging-version 2.0. Eur J Nucl Med Mol Imaging. 2015;42:328-354.

10. Coleman RE, Hillner BE, Shields AF, et al. PET and PET/CT reports: observations from the National Oncologic PET Registry. J Nucl Med. 2010;51:158-163.

11. Freudenberg LS, Hicks RJ, Beyer T. An international survey on clinical reporting of PET/CT examinations: a starting point for cross-specialty engagement. $\mathrm{J} \mathrm{Nucl}$ Med. 2019;60:480-485. 